\title{
Oncologist
}

\section{Beyond Costs and Benefits: Understanding How Patients Make Health Care Decisions}

\author{
Peter A. Ubel \\ VA Health Services Research \& Development Center for Practice Management and Outcomes Research, VA \\ Ann Arbor Healthcare System, Ann Arbor, Michigan, USA; Center for Behavioral and Decision Sciences in \\ Medicine, Ann Arbor, Michigan, USA; Division of General Internal Medicine and Department of \\ Psychology, University of Michigan, Ann Arbor, Michigan, USA
}

Key Words. Decision making • Doctor-patient communication • Psychology • Health care costs

Disclosures: Peter A. Ubel: None.

The content of this article has been reviewed by independent peer reviewers to ensure that it is balanced, objective, and free from commercial bias. No financial relationships relevant to the content of this article have been disclosed by the author or independent peer reviewers.

\begin{abstract}
Many medical decisions are "preference sensitive," where the best choice depends on the values a specific patient places on relevant outcomes. For example, a decision may require a patient to make a trade-off between a small increase in chance for survival and a large out-of-pocket expense. In such situations, patients need to understand the costs and benefits of the health care alternative, in order to decide the best course of action. However, findings from
\end{abstract}

decision science research have demonstrated that people's decisions are often influenced not only by their perceptions of these costs and benefits, but also by subtle contextual factors that trigger intuitive and emotional decision processes. In this article, I describe some of these contextual factors, and lay out the challenges these factors raise for clinicians' efforts to help their patients make informed decisions. The Oncologist 2010;15(suppl 1):5-10

\section{INTRODUCTION}

In recent years, exciting new treatments have entered oncology practice, treatments that have increased survival rates among people with life-threatening cancers. Unfortunately, some of these treatments are extremely expensive, requiring doctors and patients to decide whether the clinical benefits brought by these treatments outweigh their costs.

Many oncologists feel uncomfortable factoring these financial costs into their clinical decisions [1]. They struggle to figure out the best way to communicate about such issues with their patients. However, difficult trade-offs have long been common in oncology settings. Oncology patients have had to choose between quantity and quality of life, for example, and between short- and long-term survival. Indeed, clinicians have increasingly recognized that the "best" choice for any given oncology patient often depends on the values that specific patients place on relevant health outcomes.

Given that trade-offs have long been a critical part of oncology decision making, the arrival of expensive new on-

Correspondence: Peter A. Ubel, M.D., Center for Behavioral and Decision Sciences in Medicine, 300 North Ingalls Building, Room 7C27, Ann Arbor, Michigan 48109-5429, USA. Telephone: 734-615-8377; Fax: 734-936-8944; e-mail: paubel@umich.edu Received August 20, 2009; accepted for publication September 15, 2009. CAlphaMed Press 1083-7159/2010/\$30.00/0 doi: 10.1634/theoncologist.2010-S1-5 
cology treatments may seem, on the surface, to be just one more cost to throw into the clinical equation. By this view, oncologists should make sure their patients understand the costs and benefits of their treatment alternatives, with the costs they describe to patients encompassing not only the side effects of the treatment but also the financial impact of treatment. Armed with this broader set of information, it would seem that patients should be able to work together with their clinicians to weigh these costs and benefits, and to choose the treatment that best fits their goals.

In this article, I explain why patients' decisions are not so simple. Specifically, I explore the role that intuition and emotion play in the way people process cost and benefit information.

Experts have long recognized that health care decisions are influenced by people's emotions. But they have only recently begun to recognize that whenever people think their way through decisions, they feel their way too [2-5]. As people cogitate about the pros and cons of their decision alternatives, the affective centers of their brain also react to those same pros and cons [6]. Sometimes these two processes agree. When they do not, however, in many cases it is the affective centers that rule the day [2].

The role of affect in decision making raises fundamental challenges for cancer risk communicators. In the absence of affect, for example, oncologists could involve cancer patients in their health care decisions by taking the time to communicate the risks and benefits of their treatment alternatives, checking to make sure patients understand the information and have time to integrate that information with their individual preferences. This would be no simple task, because oncologists would need to overcome many barriers to help the patients understand their situations. But in the presence of affect, this challenge becomes even larger.

Below, I describe a number of studies that reveal some of the roles that emotion, or affect, can play in people's decisions about cancer care. (For the purposes of this article, I use the terms emotion and affect interchangeably.) In the process, I explore some of the special challenges clinicians are likely to encounter as they attempt to incorporate the financial costs of treatment into their clinical discussions.

\section{Weighing Costs And Benefits}

To illustrate the general theme of this article, consider the following hypothetical scenario, which my colleagues and I have posed to a large number of lay people and clinicians. In this scenario, we ask people to imagine that they have been diagnosed with colon cancer, and that without treatment they will die. We then describe two treatment
Table 1. Hypothetical treatment options for colon cancer

\begin{tabular}{|c|c|c|}
\hline \multirow[b]{2}{*}{ Possible outcome } & \multicolumn{2}{|c|}{ Treatment options } \\
\hline & Surgery 1 & Surgery 2 \\
\hline Cure without complications & $80 \%$ & $80 \%$ \\
\hline Cure with colostomy & $1 \%$ & \\
\hline Cure with chronic diarrhea & $1 \%$ & \\
\hline $\begin{array}{l}\text { Cure with intermittent bowel } \\
\text { obstruction }\end{array}$ & $1 \%$ & \\
\hline Cure with wound infection & $1 \%$ & \\
\hline No cure (death) & $16 \%$ & $20 \%$ \\
\hline
\end{tabular}

options to them, which we call surgery 1 and surgery 2 [7]. The risks and benefits of these two surgeries are shown in Table 1.

How should people decide between surgery 1 and surgery 2? The dominant view among decision-making experts is that people ought to make decisions like this by weighing the costs and benefits of each option-by thinking about the probability of each possible outcome and the value they place on each of these outcomes. This view is the basis of decision analysis [8], the health belief model [9], and economic theories of rationality $[10,11]$. As such, this view emphasizes explicit cognitive judgments- the rational weighing of pros and cons.

Returning to Table 1, the pros and cons of the two surgeries are clear. Both provide an $80 \%$ chance of surviving the cancer without complications. The cure rate of the two surgeries, however, differs. Surgery 2 yields a $20 \%$ death rate from cancer, whereas surgery 1 yields only a $16 \%$ death rate. The remaining $4 \%$ of the people receiving surgery 1 do not die of their cancer but, instead, survive with some kind of temporary or permanent surgical complication. The two treatments, in other words, involve a trade-off between accepting the chance for these complications and accepting a higher chance for death. The decision depends on what people think about dying from cancer versus living with either of these surgical complications.

As it turns out, most people have little difficulty saying what they think about this trade-off. When faced with a choice between dying and living with a colostomy, $>90 \%$ of the people we surveyed said they would choose to live with the colostomy. They feel even stronger about their preference for the other three surgical outcomes, compared with death. In fact, $>90 \%$ of the people we surveyed preferred each of the four surgical complications to death.

Based on these values, it seems obvious that surgery 1 is the best treatment for $>90 \%$ of people. And yet in our stud- 
ies we have found that, when given this choice, a majority of people choose surgery 2 [12].

Why do they make this choice?

\section{DECISION BIASES AND EMOTION}

Until recently, most decision scientists assumed that errors like these had to be caused by cognitive limitations that led to biased decision making [13]. In fact, over the past 40 years, researchers in the field of judgment and decision making have been documenting the many different ways that people's judgments and decisions fall short of rational ideals. In particular, researchers have demonstrated that people are not good at generating accurate probability estimates. Their estimates are susceptible to phenomena such as anchoring biases [14] and availability biases [15]. And they have learned that people's cognitive capacities are limited, in ways that make it difficult for them to weight complex costs and benefits in a rational manner [16]. They cannot "do the math" well enough to factor in all the decision-relevant trade-offs [17].

But do these cognitive limitations explain people's choices in our hypothetical colon cancer decision? Probably not. In a series of studies, my research team has tested for potential cognitive biases that could explain people's choices in this scenario [12]. For example, we tested whether people were confused by our colon cancer scenario, and discovered that most people understand the scenario quite well. In fact, we even found that physicians choose surgery 2 almost as often as the general public (unpublished data). Why the strange choices, then? I expect that people feel that surgery 2 is better for them, even though their own preferences tell them that surgery 1 is the best choice. Feeling and thinking do not always point people in the same direction. For example, Denes-Raj et al. [18] gave people a chance to win money by picking a jelly bean from one of two bowls, offering them $\$ 1$ if they chose a red jelly bean. The first bowl contained nine red beans in 100 and was labeled (accurately) as having 9\% red beans. The second bowl contained one red bean in 10 and was labeled as having $10 \%$ red beans. Many people in this study reported knowing that the second bowl gave them the best chance for winning, but feeling like the first bowl gave them a better chance because it contained a larger number of red beans. And many people were compelled by these feelings to choose from the first bowl.

We have encountered this type of reasoning frequently when discussing our colon cancer scenario. Many people tell us that they know that the first surgery is better than the second, but feel that they should still choose surgery 2, so that they won't have to deal with the possibility of experiencing a surgical complication. After all, descriptions of things like having a colostomy or a wound infection are affect-rich, evoking strong feelings of fear or disgust. It is hardly surprising that the prospect of experiencing these conditions evokes an avoidance reaction. But this is only one example of a much more general phenomenon. As I demonstrate in the next few studies, risk information is rarely received dispassionately, but is usually processed by people in affective and intuitive ways, too. Risks create feelings.

\section{The Hazards of Education}

Consider how a seemingly innocuous pretest influences the "feel" of breast cancer risk [19]. We asked a group of women to estimate the average woman's lifetime risk of breast cancer. According to the National Cancer Institute, the actual risk is approximately $13 \%$. However, most women in our study estimated that the risk was much higher than this figure, with a mean estimate of $41 \%$. Concerned about such misestimates, which have been demonstrated in numerous studies [19-23], health care researchers have developed communication interventions, designed to improve people's risk perceptions. In one such intervention, Lerman et al. [20] asked women to estimate their lifetime risk of being diagnosed with breast cancer, then educated these women about their actual risk. At the end of this educational intervention, the researchers determined that women were significantly more accurate in reporting their own risk. The educational intervention, in other words, had worked. But unfortunately, at the same time, they discovered that many women had lost interest in receiving mammograms [20].

I believe that women in this study lost interest in mammography because the $13 \%$ figure felt low to them, compared with the numbers they estimated. In fact, my colleagues and I conducted a study exploring whether the pretest, itself, influenced how women respond to the communication intervention. We randomized participants into one of two groups, one of which was asked to estimate the average woman's risk of breast cancer before receiving the $13 \%$ figure and a second that received the $13 \%$ figure without being asked to make any kind of estimate.

We found that women's reactions to the $13 \%$ figure differed significantly across the two groups. The first group, after overestimating the risk of breast of cancer, was more likely to say that the $13 \%$ figure made them feel "relieved," and was also more likely to say that the risk struck them as "low" (Table 2). By contrast, the second group was not particularly relieved by this information. In fact, collectively, they exhibited what is known as a "hindsight bias" [24] on average, they indicated that the $13 \%$ figure was just about what they would have guessed it to be.

This study demonstrates two important findings. First, 
Table 2. Effect of estimating the average woman's lifetime breast cancer risk on reactions to actual risk information

\begin{tabular}{lll}
\hline & $\begin{array}{l}\text { Estimate } \\
\text { group }\end{array}$ & $\begin{array}{l}\text { No estimate } \\
\text { group }\end{array}$ \\
\hline Feel relieved about risk & $40 \%$ & $19 \%$ \\
Risk perceived as low & $43 \%$ & $16 \%$ \\
\hline
\end{tabular}

guessing the risk influenced how women responded to the risk information. This raises important concerns for studies of communication interventions and decision aids. If researchers conduct pretests prior to their interventions, they may alter the way people perceive subsequent information. Second, the $41 \%$ figure wasn't already in women's heads when they were asked to make the estimates. If it had been, the act of guessing would not have influenced the first group's subsequent reactions to the information, nor would the second group have been susceptible to hindsight bias. Instead, the pretest forced women to come up with a numerical estimate, and this estimate then influenced their subsequent reaction to the actual risk information.

The researchers designing the breast cancer communication intervention were treating $13 \%$ like it was simply a number, indicating that 13 in 100 women develop breast cancer. By this view, $13 \%$ should be treated as being a lower risk than $15 \%$ and a higher risk than, say, $10 \%$. But this view places too much emphasis on the cognitive meaning of $13 \%$, and underemphasizes the affective or intuitive meaning of the number.

\section{RISK: A BASIS FOR COMPARISON, NOT JUST A NUMBER}

When people receive information about cancer risks, they do not simply encode the numbers into a mathematical algorithm. They pull meaning out from the numbers, stamping the information with affective or intuitive labels such as "high versus low" or "something to be worried about versus something to be relieved about." Which meaning people take away from risk information, however, can depend on what other statistics they know.

When a patient learns that a procedure has a $28 \%$ success rate, that information is initially very hard to evaluate. Is $28 \%$ good or bad? Most patients lack the domain-specific knowledge to know. But if you tell them that an alternate procedure has a $35 \%$ success rate, all of a sudden the $28 \%$ rate won't feel very good at all. In fact, providing such additional contextual information not only changes how people feel about their alternatives, it can change what they choose to do [25].
To illustrate this point further, consider a study my colleagues and I conducted that demonstrated that the way people encode information about risk can depend on whether they believe their own risk is higher or lower than average [26]. We asked women to imagine that they had a $6 \%$ risk of developing breast cancer over the next 5 years. (We chose the $6 \%$ figure because it was the average risk of women who had been enrolled in the P-1 Trial, a study that showed that tamoxifen can reduce the risk of experiencing a first breast cancer [27].) We also asked women to imagine that they could take a pill that would cut their risk in half, to $3 \%$. We then described several potential side effects of this hypothetical pill: a $0.2 \%$ chance for endometrial cancer, a $0.5 \%$ chance for heart attack or stroke, and a $15 \%$ chance for experiencing hot flashes or vaginal discharge. We asked women how interested they would be in this pill, how worried they would be about their chance for breast cancer, and how effective they thought the pill would be.

Whereas every woman who participated in our study received identical risk information-all were told their own 5 -year risk was 6\%-we designed the study to test whether the way women felt about both breast cancer and the prevention pill would change if they were given hypothetical information suggesting that their $6 \%$ risk was above or below average. We told some participants that the average risk of breast cancer over 5 years was $3 \%$, and another group that the average was $12 \%$.

Women's perceptions of breast cancer and of the prevention pill were significantly influenced by this comparative information. Those in the $3 \%$ group, who now felt at above average risk, were more worried about their own risk of breast cancer than the $12 \%$ group, who felt at below average risk. Indeed, the above average group also ended up more interested in taking the pill than the other group, and more convinced about the effectiveness of the pill.

The choice facing these women was to decide whether a $3 \%$ absolute reduction in the risk for breast cancer is a large enough benefit to justify the risks of this pill. The comparative information we provided did nothing to change either the risks or the benefits of the pill. And yet, the comparative information significantly influenced how women felt both about breast cancer and about the risks and benefits of the pill. The $6 \%$ figure was not just a number, in other words. It was a piece of information that people coded as above or below average.

Paul Windschitl, a psychologist at the University of Iowa, has conducted a large number of studies that have illustrated the distinction between what people believe about risks and what they intuit about the risks [28, 29]. He contends that people's beliefs about the numeric probability of an event, like the $6 \%$ risk for breast cancer over 5 years, are 
only part of how people perceive the risk. There is also "a more intuitive and non-analytic component to uncertainty that is not necessarily well represented in a numeric subjective probability response but can be an important mediator of decisions and behavior" [29].

Providing comparative risk statistics is not the only way to influence the intuitive meanings patients draw from risk statistics. For example, in a study examining prenatal genetic screening decisions [30], we discovered that the seemingly innocuous practice of labeling a screening test result as "negative" or "positive" significantly changed people's risk perceptions and their decision making about amniocentesis.

\section{MONEY TALK}

I have tried to give a few examples to show the critical role that intuition and emotions play in people's health care decisions. Giving patients information about the costs and benefits of health-related choices won't always lead to optimal decisions. Clinicians need to be aware of the subtle contextual factors that can influence the way people perceive the costs and benefits of health care interventions, and thereby influence the health care choices people make.

Consider the costs and benefits of expensive new oncology treatments. If patients were completely rational, then clinicians could simply inform patients about the costs and benefits of such treatments-with costs broadly construed to include out-of-pocket financial costs-and patients could decide whether the benefits of such treatments outweighed the costs. But human beings are not completely rational. Therefore, clinicians need to be aware of the psychological forces that could lead patients to make suboptimal decisions.

For example, clinicians need to consider the typical social context of a doctor-patient interaction. The doctor enters into this interaction with much more information than the patient, and with much more experience facing the kinds of choices that this patient must consider. The patient may be in a high state of emotional arousal. A cancer diagnosis can be a shocking thing to learn about. The patient may also be reaching the point of cognitive saturation-he may be feeling overwhelmed by the information he needs to consider in making an informed choice.

Add to this challenging mix a dose of financial reality, and things can get quite dicey. You see, financial conversations are not a routine part of most clinical encounters. Outside settings like plastic surgery, most patients do not often talk about the financial cost of interventions with their providers. Indeed, it is rare for doctors to talk about the cost of care when patients face life-threatening illnesses. I expect, for example, that many clinicians would feel uncomfortable talking with patients about whether the benefits of an intensive care unit stay are substantial enough to justify the steep financial cost of such a treatment. Indeed, it is already difficult for many clinicians to find the time to help patients understand the medical risks and benefits of their treatment alternatives. Including financial costs in such discussions simply makes matters even more challenging.

Financial costs might be especially difficult for patients to incorporate into clinical decisions, because such costs are so hard to compare with health-related costs and benefits. All decisions, of course, require people to make trade-offs. But some trade-offs are harder to make than others. For example, if patients face a trade-off between short-term mortality and long-term mortality, they are comparing "apples to apples." They can decide what risk of death in the next year they are willing to accept from aggressive treatment in order to increase their chance for long-term survival.

Patients' decisions become more difficult when they are asked to compare apples to oranges, metaphorically speaking of course. For instance, some decisions require patients to make trade-offs between length of life and health-related quality of life.

Now consider making a choice between money and life. How much money would you spend for, say, a 5\% increase in your chance of surviving for 5 years? I expect that most people have a very hard time making this kind of decision. They cannot draw upon a great deal of experience in their own lives where they have traded off money versus survival. In addition, it is hard to compare money to survival. If choosing between length of life and quality of life is like comparing apples to oranges, then choosing between money and survival is like comparing apples to cell phones-they don't even feel like they belong in the same general category.

I think of money and cancer as a triple whammy: just when patients' emotions are highest (new cancer diagnosis!), they are forced to talk about money for the first time with their physician, and in the process must compare their financial security with their chance for long-term survival.

\section{Conclusion}

Physicians are increasingly recognizing that many medical decisions are not purely medical decisions, but hinge on patient preferences. This recognition of patients' preferences has raised the challenge of finding ways to communicate the costs and benefits of treatments in ways that patients not only comprehend, but can also integrate with their own values to help the clinician determine what treatment choice is best for that given patient.

The existence of extremely expensive new oncology 
treatments has created a new challenge for this paradigm of shared decision making. Clinicians can expect to struggle through this new challenge. In doing so, they should remember that helping patients understand the costs and benefits of treatments is a crucial part of shared decision making. But they also need to recognize that patients' emotions and intuitions will play a large role in the ultimate decision that they make. Being aware of such emotions is a crucial early step for clinicians to help their patients figure out what is in their own best interests.

\section{REFERENCES}

1 Neumann P, Palmer J, Abrahams D et al. Struggling with expensive new cancer therapies: A national survey of oncologists. Health Aff 2009 (in press).

2 Loewenstein GF, Weber EU, Hsee CK et al. Risk as feelings. Psychol Bull 2001;127:267-286.

3 Finucane ML, Alhakami A, Slovic P et al. The affect of heuristic judgments of risks and benefits. J Behav Decis Mak 2000;13:1-17.

4 Damasio AR. Descartes' Error: Emotion, Reason, and the Human Brain. New York: G. P. Putnam's Sons, 1994:1-312.

5 Rottenstreich Y, Hsee CK. Money, kisses, and electric shocks: On the affective psychology of risk. Psychol Sci 2001;12:185-190.

6 LeDoux JE. The Emotional Brain: The Mysterious Underpinnings of Emotional Life. New York: Simon \& Schuster, 1996:1-384.

7 Ubel PA. Is information always a good thing? Helping patients make "good" decisions. Med Care 2002;40(9 suppl):V39-V44.

8 Ubel PA, Loewenstein G. The role of decision analysis in informed consent: Choosing between intuition and systematicity. Soc Sci Med 1997;44: 647-656.

9 Strecher VJ, Rosenstock IM. The health belief model. In: Glanz K, Lewis FM, Rimer BK, eds. Health Behavior and Education: Theory, Research, and Practice, Second Edition. San Francisco: Jossey-Bass, 1997:41-59.

10 Baron J. Thinking and Deciding, Second Edition. New York: Cambridge University Press, 1994:1-576.

11 Ubel P. Free Market Madness: Why Human Nature is at Odds with Economics-and Why it Matters. Boston: Harvard Business Press, 2009:1240 .

12 Amsterlaw J, Zikmund-Fisher BJ, Fagerlin A et al. Can avoidance of complications lead to biased healthcare decisions? Judgm Decis Mak 2006;1: $64-75$.

13 Kahneman D, Slovic P, Tversky A, eds. Judgment Under Uncertainty: Heuristics and Biases. Cambridge: Cambridge University Press, 1982:1-544.

14 Tversky A, Kahneman D. Judgment under uncertainty: Heuristics and biases. Science 1974;185:1124-1131.

15 Tversky A, Kahneman D. Availability: A heuristic for judging frequency and probability. Cogn Psychol 1973;5:207-232.

16 Simon H. Bounded rationality. In: Eatwell J, Milgate M, Newman P, eds. The New Palgrave: Utility and Probability. New York: W. W. Norton \& Company, 1990:15-18.
17 Fagerlin A, Ubel PA, Smith DM et al. Making numbers matter: Present and future research in risk communication. Am J Health Behav 2007;31(suppl 1):S47-S56.

18 Denes-Raj V, Epstein S, Cole J. The generality of the ratio-bias phenomenon. Pers Soc Psychol Bull 1995;21:1083-1092.

19 Fagerlin A, Zikmund-Fisher BJ, Ubel PA. How making a risk estimate can change the feel of that risk: Shifting attitudes toward breast cancer risk in a general public survey. Patient Educ Couns 2005;57:294-299.

20 Lerman C, Lustbader E, Rimer B et al. Effects of individualized breast cancer risk counseling: A randomized trial. J Natl Cancer Inst 1995;87:286292.

21 Croyle RT, Lerman C. Risk communication in genetic testing for cancer susceptibility. J Natl Cancer Inst Monogr 1999;25:59-66.

22 Mouchawar J, Byers T, Cutter G et al. A study of the relationship between family history of breast cancer and knowledge of breast cancer genetic testing prerequisites. Cancer Detect Prev 1999;23:22-30.

23 Durfy SJ, Bowen DJ, McTiernan A et al. Attitudes and interest in genetic testing for breast and ovarian cancer susceptibility in diverse groups of women in western Washington. Cancer Epidemiol Biomarkers Prev 1999; 8:369-375.

24 Fischhoff B. Hindsight is not equal to foresight: The effect of outcome knowledge on judgment under uncertainty. J Exp Psychol Hum Percept Perform 1975;1:288-299.

25 Zikmund-Fisher BJ, Fagerlin A, Ubel PA. "Is 28\% good or bad?" Evaluability and preference reversals in health care decisions. Med Decis Making 2004;24:142-148.

26 Fagerlin A, Zikmund-Fisher BJ, Ubel PA. "If I'm better than average, then I'm OK?": Comparative information influences beliefs about risk and benefits. Patient Educ Couns 2007;69:140-144.

27 Fisher B, Costantino JP, Wickerham DL et al. Tamoxifen for prevention of breast cancer: Report of the National Surgical Adjuvant Breast and Bowel Project P-1 Study. J Natl Cancer Inst 1998;90:1371-1388.

28 Windschitl PD, Martin R, Flugstad AR. Context and the interpretation of likelihood information: The role of intergroup comparisons on perceived vulnerability. J Pers Soc Psychol 2002;82:742-755.

29 Windschitl PD. Judging the accuracy of a likelihood judgment: The case of smoking risk. J Behav Decis Mak 2002;15:19-35.

30 Zikmund-Fisher BJ, Fagerlin A, Keeton K et al. Does labeling prenatal screening test results as negative or positive affect a woman's responses? Am J Obstet Gynecol 2007;197:528.e1-e6. 